\title{
A Fall and Near-Fall Assessment and Evaluation System
}

\author{
Basran $^{4}$, Seok-Bum Ko ${ }^{1}$ and Carl McCrowsky ${ }^{1}$ \\ ${ }^{I}$ Department of Electrical and Computer Engineering, University of Saskatchewan, Canada \\ ${ }^{2}$ Department of Mechanical Engineering, University of Saskatchewan, Canada \\ ${ }^{3}$ School of Physical Therapy, University of Saskatchewan, Canada \\ ${ }^{4}$ Division of Geriatric Medicine, University of Saskatchewan, Canada
}

Anh Dinh ${ }^{1}$, Yang $\mathrm{Shi}^{2}$, Daniel Teng*, ${ }^{*}$, Amitoz Ralhan ${ }^{1}$, Li Chen $^{1}$, Vanina Dal Bello-Haas ${ }^{3}$, Jenny

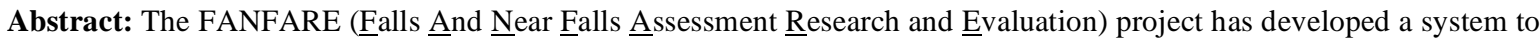
fulfill the need for a wearable device to collect data for fall and near-falls analysis. The system consists of a computer and a wireless sensor network to measure, display, and store fall related parameters such as postural activities and heart rate variability. Ease of use and low power are considered in the design. The system was built and tested successfully. Different machine learning algorithms were applied to the stored data for fall and near-fall evaluation. Results indicate that the Naïve Bayes algorithm is the best choice, due to its fast model building and high accuracy in fall detection.
\end{abstract}

Keywords: Fall detection, near-fall data collection, wearable device, machine learning, fall classification, wireless communications.

\section{INTRODUCTION}

Falls in the elderly present a major challenge for public health [1]. Falls not only cause disabling fractures but also has dramatic psychological consequences, which reduce the independence of a person. Fall can be described in a common sense as a rapid change from the upright/sitting position to the reclining or almost lengthened position in an uncontrolled movement. The postural stability of the movement is affected by complex internal neural and muscular models [2]. Older adults who have experienced frequent near fall events or repeated falls are typically assessed using a documentation method to determine fall risk, where fall events and the circumstances surrounding fall events are evaluated through questioning and self-report systems using questionnaires, fall diaries or phone calls. These documentation methods have advantages and disadvantages in terms of accuracy, costs and time commitments [3]. One notable concern to all the documentation methods are their associated problems of subjectivity. Due to the limited accuracy with respect to recall, the reported circumstances surrounding falls are often incomplete or inaccurate. In addition, falls are more than likely underreported through the documentation methods. Near-fall events are particularly difficult to determine, because often older adults do not recognize the events themselves or do not recognize the significance of the nearfall events. On the other hand, fall risk factors are determined with physical examination, which includes evaluation of posture, balance, gait, visual acuity, sensation, vital signs, and activities of daily living. Although useful, a physical examination only evaluates the risk factors at one particular

*Address correspondence to this author at the University of Saskatchewan, 57 Campus Drive, Saskatoon, SK S7N5A9, Canada; Tel: (306) 966-2889; Fax: (306) 966-5407; E-mail: daniel.teng@usask.ca point in time, and is based on a short period of observation. Suspected causes of falls or near-falls, such as cardiac arrhythmias and changes in blood pressure, require further tests. Unfortunately, this causes delay in diagnosis. More importantly, the tests are conducted in isolation of each other and in isolation of multiple compounding factors that may be occurring during an actual near-fall or fall event. For example, a change in blood pressure alone may not lead to a fall, but when combined with poor balance (detected as increased sway) may result in a fall.

Falls are multi-factorial in nature; therefore assessment and detection methods need to be multi-factorial. Numerous fall detection methods have been proposed in the past [4]. Newell [5] approached the fall detection with monitoring the change in the patterns of inactivity. Sixsmith [6] used an array of infrared detectors for fall monitoring. A method of evaluating the characteristics of postural transition (PT) and their correlation with falling risk in elderly people is described in [7]. More recently, accelerometry has been seen as a practical, inexpensive and reliable method for the monitoring of postural motion as well as for the detection and prediction of falls. A wearable device featuring three monoaxial accelerometers and three gyroscopes to assess fall risks is proposed in [8]. Lee [9] proposed an accelerometer sensor module and associated wireless sensor network to assess fall risks. Motivated by the need for more accurate fall and nearfall detection in the medical community, this fall detection and data collection system is designed to capture physiological activities of a patient for fall and near-fall analysis or monitoring. The collected data are extremely useful for medical personnel for pre-fall and post-fall analysis. The data can be used to determine the fall pattern of a patient. The system also provides real-time monitor on the elderly people for fall prevention. For instance, a combination of fall 
parameters such as fall backward, forward, heart rate, etc. can be used for the prevention of future falls on that person by providing a fall warning signal.

\section{SYSTEM DESIGN}

The fall detection and data collection system is shown in Fig. (1) which includes a networked computer, a wireless receiver connected to the computer and a wearable device which is worn by a patient. The wearable device is called a sensor node which houses the sensors and sends the fall related data wirelessly to the receiver.

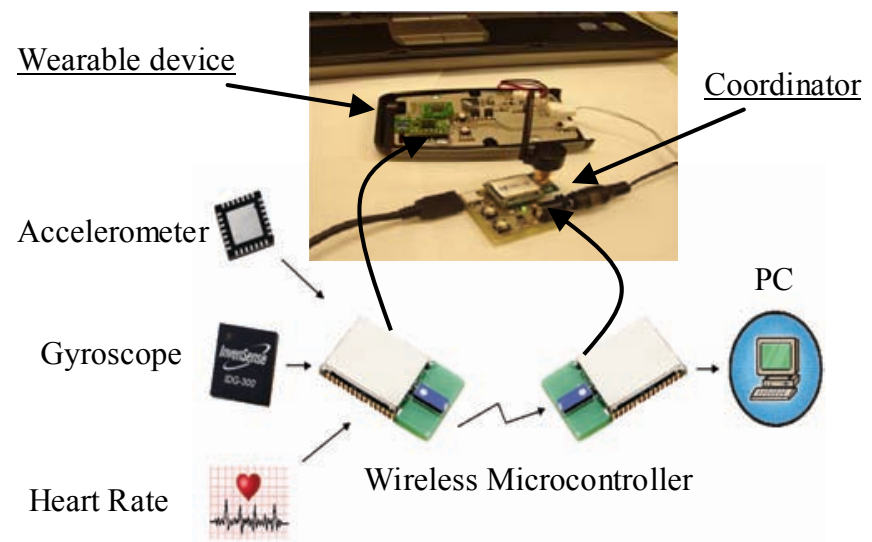

Fig. (1). The fall detection and data collection system consists of a network connected computer and a wireless sensor network comprising a coordinator and a sensor node.

The system detects falls by measuring, collecting, and analyzing fall parameters including postural sway in 3-D using a 3-axis acceleromter and a 2-axis gyroscope and a heart rate sensor. The on-board microcontroller with ZigBee ready communication protocol is used for controlling the sensors and also preprocessing collected data before sending it to the receiver. Different fall detection algorithms can be programmed into the wearable device to provide warning signal to the user to alert a possible fall in order to take fall prevention measures.

\section{Sensor Node on the Wearable Device}

Fig. (2) provides a closer look at the wearable device. This device (i.e., a sensor node) comprises of a high precision acclerometer sensor (ST LIS3LV02DQ), a gyroscope

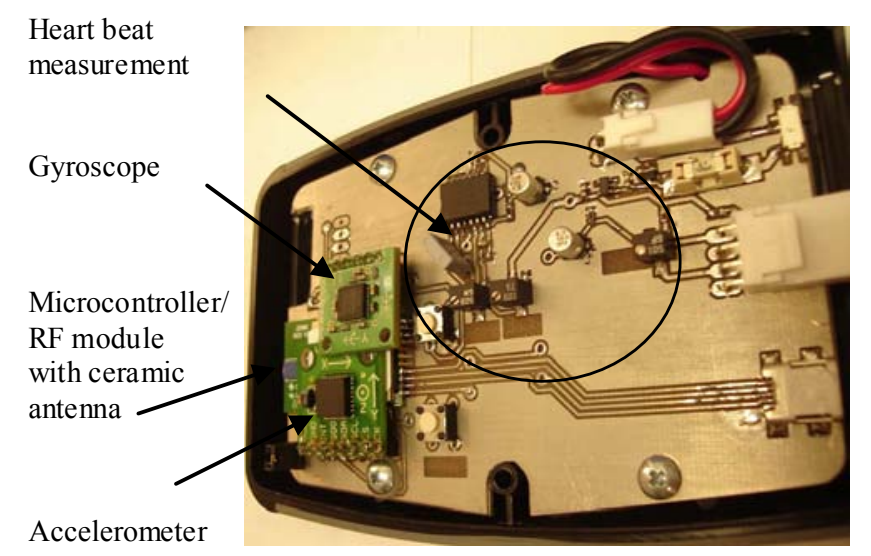

Fig. (2). The wearable device.
(InvenSense IDG-300), a heart rate sensor, an RF-ready microcontroller (Jennic 5139-Z01) and a rechargeable battery mounted underneath the circuit board (not shown in the figure).

The LIS3LV02DQ is a three-axis, 12-bit digital output linear accelerometer that includes a sensing element and an $\mathrm{I}^{2} \mathrm{C} / \mathrm{SPI}$ serial interface to take the acceleration information from the sensing element and to provide the measured acceleration signals to the external world. The sensor, operating on a $2.16 \mathrm{~V}-3.6 \mathrm{~V}$ supply, has a user selectable full scale of $\pm 2 \mathrm{~g}$ or $\pm 6 \mathrm{~g}$. The device may be configured to generate an inertial wake-up/free-fall interrupt signal when a programmable acceleration threshold is crossed at least in one of the three axes. This feature is very useful in this application because: a) the wearable device can save power by putting the sensor to sleep mode and $b$ ) the sensor can be programmed to detect a fall for a certain threshold to be determined by a particular individual after pre-fall analyses.

The dual-axis gyro is a MEMS device operating at a single supply voltage of 3.0-3.5V. The sensor provides analog outputs of $\mathrm{X}$ and $\mathrm{Y}$ rates with a full scale of $\pm 500 \% \mathrm{sec}$. The output voltages of the rotations are connected to the onboard analog-to-digital (ADC) of the microcontroller/RF module. One of the disadvantages of the gyro is its output drifting due to the nature of the gyro. Further filtering is required to correct the error as a result. The sensor also consumes power continuously due to its long wake-up time. Both the accelerometer and the gyro have very high shock survivability of at least $500 \mathrm{~g}$. The combination of accelerometer and gyroscope provides a better postural activity measurement as proved in the testing results.

The heart rate sensor circuit is based on the pulse plethysmograph (PPG) method. PPG is an optic signal related to the volumetric pulsations of blood in tissue, which in turn is related to heart beat. As shown in the circuit block diagram of Fig. (3), the circuit uses an infrared (IR) LED to send a light through an earlobe to an IR transistor. The diode/transistor pair with appropriate bias voltage and current detects the blood flow through the earlobe. The amplification and filtering convert the small current flowing through the transistor into a heart beat signal.

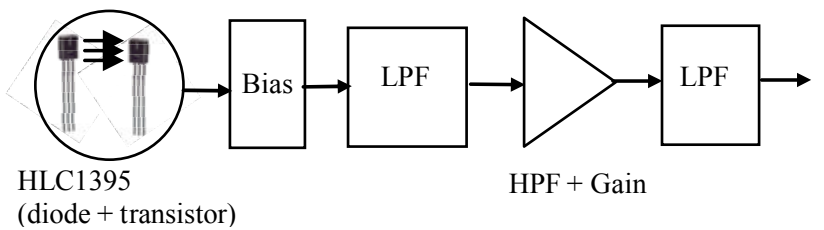

Fig. (3). Heart beat sensor block diagram.

JN5139 is a RF-ready microcontroller module made by Jennic [10]. This low-cost, off-the-self module has a microcontroller and wireless communications in a single unit to provide the necessary resource to design a wireless sensor network with multiple sensor nodes using the ZigBee protocol. This module also includes a built-in ceramic antenna. One of the important features is that the unit can be put into the sleep mode which requires only $3 \mu \mathrm{A}$ of current to run the active sleep timer. Sleep mode is used extensively in this system to reduce power consumption of the sensor node 
which is running on a rechargeable battery. The 32-bit RISC CPU in this module provides the ability to perform tasks such as processing data, controlling the SPI accelerometer, and performing ADC conversion of the heart rate sensor.

\section{The Coordinator for Receiving Data}

JN5139 can also be programmed to perform the coordination function. As a coordinator, JN5139 can be connected to a ceramic antenna or an external antenna to extend the transmission range as shown in Fig. (4). The combination of ceramic antenna in the transmitter and external antenna at the receiver provides a tested transmission range of at least $15 \mathrm{~m}$ with non-line-of-sight.

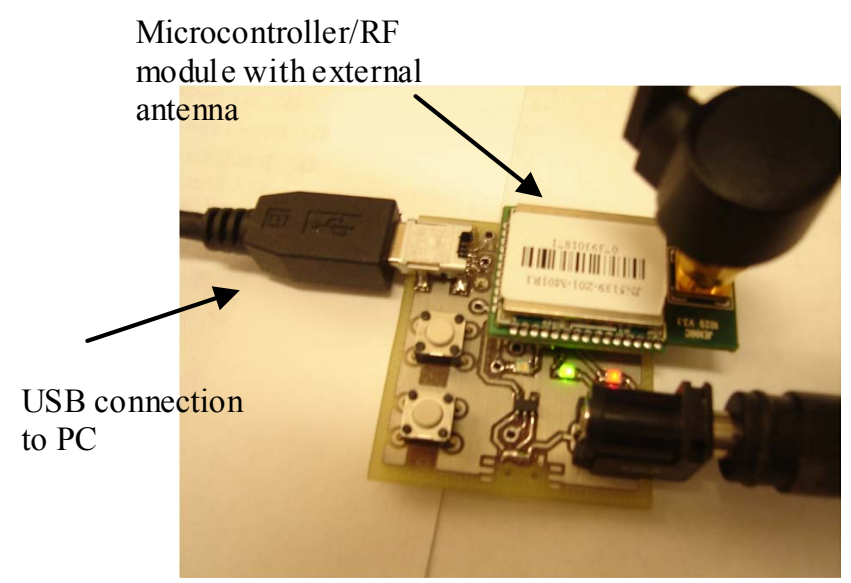

Fig. (4). The coordinator.

As shown in Fig. (1), the coordinator is the main controller connected to a computer. The coordinator communicates directly to the sensor node. Through the coordinator, the computer receives physiological activity data sent by the wearable device. The computer is also used for storing data in CSV format for easy retrieval. The time stamped data can be retrieved and analyzed using any fall detection analyzing methods. One such method is the data classification using support vector machines described in [11]. An algorithm to detect the fall using acceleration data has developed by Hwang, et al. in [12] while Luo and Hu applied a 3D body motion model to represent acceleration vector in $3 \mathrm{D}$ space to detect the fall using a three stage dynamic process [13]. The computer also displays the $\mathrm{X}, \mathrm{Y}$ and $\mathrm{Z}$ coordinates of the accelerometer, the $\mathrm{X}$ and $\mathrm{Y}$ axes of the gyroscope, as well as the heart beat signal for real time monitoring.

\section{THE SOFTWARE}

This system is a hardware/software co-design in which the software must be programmed into the microcontroller in the Jennic module. Fig. (5a) and (5b) show the flow charts for the sensor node and for the coordinator, respectively. Upon startup, the sensor node initializes all the required subsystems and begins searching for a network (which is initialized by the coordinator). When the sensor node finds a coordinator, it will join the network, turn on the sensors, and begin the main loop. The loop checks to ensure that the battery voltage is good, then reads the sensors and sends the data to the coordinator. The Jennic module then sleeps for $100 \mathrm{~ms}$, or until the next accelerometer data sample is ready, which takes $25 \mathrm{~ms}$ for a sampling rate of 40 samples/second. If the battery voltage is low, the module will turn off the sensors to save power, and emit a pulse with the buzzer alerting the user to recharge the battery. When the voltage drops to a level where there is a risk of damaging the Lithium Polymer cell, the sensor node will turn itself off and no longer transmit data. On startup, the coordinator initializes itself and sets up the network. When a sensor joins, the coordinator will receive data from that sensor and forward it to the computer.

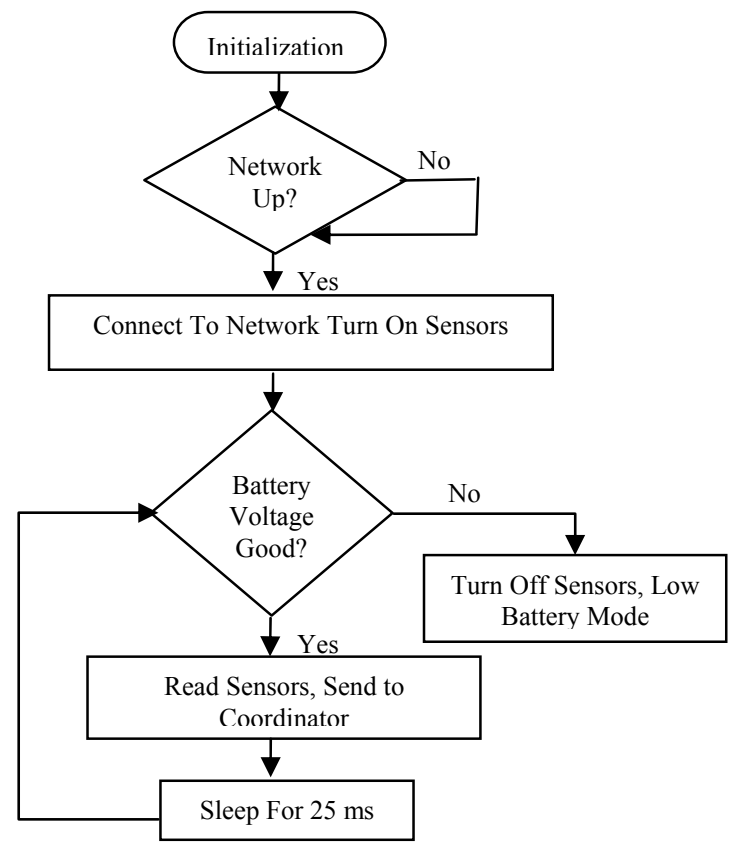

(a)

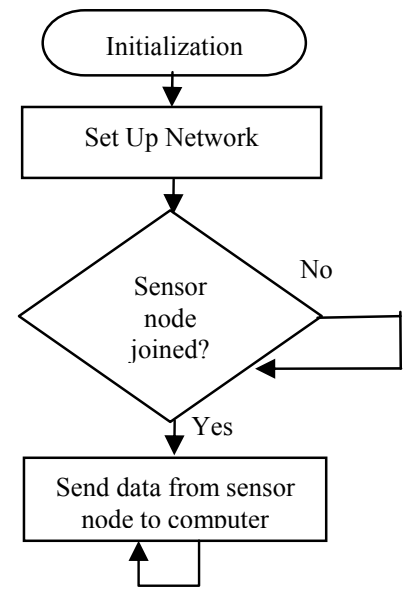

(b)

Fig. (5). Software flowchart (a) Sensor node and (b) Coordinator.

\section{SYSTEM TESTING}

One of the wearable devices was trapped on the thorax of a healthy person to test the system functionalities including data storage, display and analysis. Fig. (6) shows an example of the data stored with time stamped. The sampling rate is 40 samples per second. Data are continuously stored in a file in CSV format upon starting the system. In this sample, the first 
column lists the time of the computer receives data from the coordinator. The next 3 columns contain $\mathrm{X}, \mathrm{Y}$, and $\mathrm{Z}$ values of the accelerometer. The fifth and sixth column have $X$ and $\mathrm{Y}$ axis data from the gyroscope and the last column contains digital values of the heart beat signal. The physicians and researcher can mine the data for the required information. The size of the data file is determined by the data polling rate and the number of days to be observed, and users can name the file.

\begin{tabular}{|c|c|c|c|c|c|c|c|}
\hline & A & $\mathrm{B}$ & C & D & $E$ & $\mathrm{~F}$ & G \\
\hline 1 & 1:27:38 PN & 0.566 & -0.343 & -0.683 & 297.949 & 324.615 & 987 \\
\hline 2 & 1:27:38 PN & 0.566 & -0.343 & -0.683 & 297.363 & 326.96 & 992 \\
\hline 3 & 1:27:38 PN & 0.554 & -0.343 & -0.703 & 296.484 & 324.908 & 986 \\
\hline 4 & 1:27:38 PN & 0.563 & -0.349 & -0.706 & 298.828 & 325.202 & 986 \\
\hline 5 & 1:27:38 PN & 0.557 & -0.343 & -0.709 & 298.535 & 324.322 & 988 \\
\hline 6 & 1:27:38 PN & 0.557 & -0.34 & -0.706 & 299.707 & 325.202 & 990 \\
\hline 7 & 1:27:39 PN & 0.557 & -0.349 & -0.698 & 299.707 & 324.615 & 984 \\
\hline 8 & 1:27:39 PN & 0.554 & -0.352 & -0.709 & 295.897 & 324.615 & 988 \\
\hline 9 & 1:27:39 PN & 0.574 & -0.349 & -0.721 & 299.707 & 324.908 & 988 \\
\hline 10 & 1:27:39 PN & 0.542 & -0.352 & -0.709 & 309.963 & 322.857 & 988 \\
\hline 11 & 1:27:39 PN & 0.542 & -0.352 & -0.709 & 303.223 & 323.443 & 986 \\
\hline 12 & $1.27 \cdot 39 \mathrm{PN}$ & $\cap 5 \Delta ?$ & $-\cap 349$ & $-\cap 712$ & 299121 & 327857 & 983 \\
\hline
\end{tabular}

Fig. (6). Data storage includes time stamped, accelerometer data $(\mathrm{x}, \mathrm{y}, \mathrm{z})$, gyroscope data $(\mathrm{x}, \mathrm{y})$, and heart beat signal.

Fig. (7) is a screen captured of the display for the threeaxis accelerometer, the rotational data from the dual-axis gyros and the heart beat signal. The data display is for the random and sometime rigorous movements of the end device.

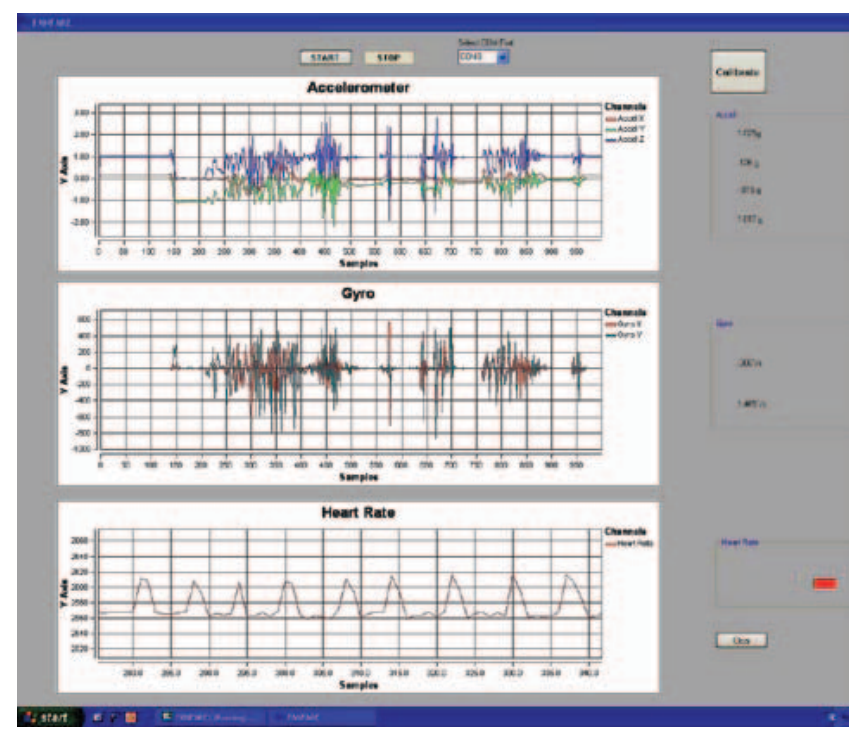

Fig. (7). Sensor node display.

The sensor node is programmed to spend most of its time in sleep mode to conserve energy unless the data are transmitted which draws a current of $30 \mathrm{~mA}$. Test results on a fullcharge battery showing the wearable device can properly operate for 70 hours. The gyroscope and the heart rate sensor must consume power continuously while the accelerometer can be put into sleep mode if desired. When the battery volt- age falls below $2.1 \mathrm{~V}$, the gyroscope stops functioning while the other parts of the sensor node continue operating.

\section{FALL DETECTION ANALYSIS}

Machine learning involves providing a training file to the learning algorithm in which the data are correctly classified. The machine then learns the patterns involved in the data and can automatically classify future situations. More accurate classification techniques ensure lesser number of false alarms and fewer undetected falls. Selection of a suitable classification algorithm is the first and the most important step in solving any classification problem. Since all classification algorithms behave differently towards different type of data, depending upon the number of attributes and the nature of outliers among other factors, a comparison of different algorithms applied to a particular data set is useful in selecting the best algorithm for the task [14]. As will be described below, this fall detection analysis applied five different classification algorithms to accelerometer and gyroscope data collected from the designed fall assessment and evaluation system.

\section{Data Collection}

As described previously, the data for the study was collected using the device shown in Fig. (1). The data consists of 3582 data items distributed in 597 rows and 6 columns. Three columns represent the acceleration in $\mathrm{X}, \mathrm{Y}$ and $\mathrm{Z}$ axis. Two columns represent the data from Gyroscope in $X$ and $Y$ axis. The entire data set is used for the training purpose and 10 -fold cross validation technique is used to test the generated model.

\section{WEKA}

Waikato Environment for Knowledge Analysis or in short WEKA [15] is used in this fall detection analysis. WEKA is a Java based data mining software developed at the University of Waikato, New Zealand and is available as a free software under GNU public license. It supports different data mining tasks such as data preprocessing, clustering, classification and regression. It is frequently used by researchers because of its ease of use and open source environment $[14,16]$. Graphical User Interfaces contained in the software make it easy to use and better visualize the data. The data is processed as an ARFF file. The tool is capable of reading data from a number of file formats like a CSV file or a SQL database and converts the data internally to the ARFF format [17].

\section{Algorithms}

WEKA consists of a number of machine learning algorithms: Naïve Bayes, Radial Basis Function, Support Vector Machine, C4.5 and Ripple Down Rule Learner.

\section{1) Naive Bayes}

Naive Bayes is a simple probabilistic classifier. It assumes that every feature related to a class is independent of each other [18]. So the probability of occurrence of a class $C$, provided the features $F_{1}$ through $F_{N}$ is

$$
P\left(C \mid F_{1}, F_{2} \ldots F_{j}\right)=C(C) \prod_{j=1}^{N} P\left(F_{j} \mid C\right)
$$


The classifier learns the conditional probability of each attribute from the training data. Classification is done by calculating the probability of $C$ given the values of features $F_{1}$ through $F_{N}$ and then predicting the class with the highest probability value. Though the independence assumption is far reaching and often inaccurate in real world data, this method performs surprisingly well for most of the classification problems [19].

\section{2) Radial Basis Function (RBF)}

A radial basis function network is an artificial neural network which uses radial basis functions as activation functions [20]. RBF networks typically have three layers. An input layer is a hidden layer with non linear RBF function and a linear output layer. The output $\varphi$ of the network for an input $x$ is given by

$\varphi(x)=\sum_{i=1}^{N} a_{i} \rho\left(\left\|x-c_{i}\right\|\right)$

where $c_{i}$ is the center for neuron $\mathrm{i}$ and $a_{i}$ is the corresponding weight. A successful implementation of these networks requires appropriate values of the center and the weights [18].

\section{3) Support Vector Machine}

Support Vector Machine is a class of linear classifiers that simultaneously minimize the empirical classification error and maximize the geometric margin. The process involves creating a hyperplane in a n-dimensional space, that would separate two data sets with the highest margin [21]. Reaching such a hyperplane is essentially a solution of the following optimization problem:

minimize $\frac{1}{2}\|W\|^{2}$ subject to $c_{i}\left(W x_{i}-b\right) \geq 1$

where $1<i<n$ and $W$ is the vector normal to hyperplane, $b$ is the offset for vector $W$.

\section{4) $C 4.5$}

C4.5 is a type of decision tree that uses Shannon's entropy as a criterion for selecting the most discriminatory feature.

$$
\operatorname{Entropy}(S)=\sum_{i=1}^{c}-p_{i} \log _{2}\left(p_{i}\right)
$$

The entire data set is split by using any one of the features and the resultant information gain is measured. The process is repeated for every feature and the one with highest information gain is selected for splitting the data [18]. This becomes the first decision node of the tree and the process is repeated for every node until the final node or the leaves are reached.

\section{5) Ripple Down Rule Learner}

Ripple Down Rule Learner is a type of machine learning algorithm that comes under the general class of rule learners. Like all rule learners, it induces a set of rules from the data. It generates the default rule first and then the exceptions for the default rule with the least (weighted) error rate. The process is repeated until the final leaf is reached which has only one default class and no exceptions [18].

\section{RESULTS}

To measure the accuracy of the machine learning algorithms, same method is applied to all the five algorithms, i.e., the entire data set is used to train the algorithms and subsequently 10 fold cross validation method is used to test the generated classification models. In WEKA, every row of data is considered as an instance and the features in the data are known as attributes. Results of the simulation show different parameters such as correctly and incorrectly classified instances, mean absolute and root mean squared error, confusion matrix etc.

Tables 1 and $\mathbf{2}$ summarize the results of the simulation based on accuracy and error. As is evident from the results shown in the tables, the Naive Bayes algorithm provides the best performance both in terms of accuracy and the time taken to build the model. It has an accuracy of $97.3 \%$ and the model was built almost instantaneously. The lowest accuracy of $92.3 \%$ was shown by the Support Vector Machine. This model also took the longest time to build, 14.16 seconds, as compared to an average time of 4.5 seconds for all the algorithms in the experiment. The average accuracy turns out to be $94.5 \%$ with an average 564 correctly classified instances and 32 incorrectly classified instances, out of a total 597 instances. Table 3 shows the confusion matrix for simulation results using Naive Bayes algorithm. All the instance belonging to the classes Forward Fall, Lying, Standing and Backward Fall have been correctly classified. The classes Walking, Right Fall and Left Fall had some of their instances misclassified as belonging to another class. In total there were 16 misclassified instances and 581 correctly classified instances.

Table 1. Accuracy of Each Algorithms

\begin{tabular}{|c|c|c|c|}
\hline Algorithm & $\begin{array}{c}\text { Correctly Classified Instances \% } \\
\text { (value) }\end{array}$ & $\begin{array}{c}\text { Incorrectly Classified Instances } \\
\% \text { (value) }\end{array}$ & $\begin{array}{l}\text { Time taking to build model }{ }^{1} \\
\text { (second) }\end{array}$ \\
\hline Naive Bayes & $97.3(581)$ & $2.7(16)$ & 0.01 \\
\hline Support Vector Machine & $92.3(551)$ & $7.7(46)$ & 14.16 \\
\hline Radial Basis Function & $95.8(572)$ & $4.2(25)$ & 8.01 \\
\hline Ripple Down Rule Learner & $92.8(554)$ & $7.2(43)$ & 0.16 \\
\hline
\end{tabular}

${ }^{1}$ Using Intel Core ${ }^{\mathrm{TM}} 2$ Duo $1.67 \mathrm{GHz}$ processor and 2GB RAM. 
Table 2. Simulation Errors for Each Algorithms

\begin{tabular}{|c|c|c|c|c|}
\hline Algorithm & Mean Absolute Error & Root Mean Squared Error & $\begin{array}{c}\text { Relative Absolute Error } \\
(\%)\end{array}$ & $\begin{array}{c}\text { Root Relative Squared } \\
\text { Error }(\%)\end{array}$ \\
\hline \hline Naïve Bayes & 0.011 & 0.075 & 4.53 & 21.47 \\
\hline Support Vector & 0.205 & 0.304 & 83.96 & 86.77 \\
\hline Radial Basis Function & 0.012 & 0.106 & 4.99 & 30.28 \\
\hline C4.5 & 0.018 & 0.119 & 8.52 & 34.04 \\
\hline Ripple Down Rule Learner & 0.020 & 0.143 & 8.40 & 40.99 \\
\hline
\end{tabular}

Table 3. Simulation Errors for Each Algorithm

\begin{tabular}{|c|c|c|c|c|c|c|c|}
\hline $\mathbf{A}$ & $\mathbf{B}$ & $\mathbf{C}$ & $\mathbf{D}$ & $\mathbf{E}$ & $\mathbf{F}$ & G & 0 \\
\hline \hline 86 & 0 & 0 & 0 & 0 & 0 & 0 & A: Forward fall \\
\hline 0 & 82 & 0 & 0 & 0 & 0 & 0 & B: Lying \\
\hline 0 & 0 & 90 & 0 & 0 & 0 & 0 & D: Standing \\
\hline 0 & 0 & 0 & 82 & 0 & 0 & E: Walking fall \\
\hline 0 & 0 & 0 & 0 & 0 & 87 & 0 & F: Bacward fall \\
\hline 0 & 0 & 0 & 0 & 0 & 0 & 77 \\
\hline 0
\end{tabular}

\section{CONCLUSION}

Testing results prove the system successfully senses, collects and stores fall related data. Using low power devices and clever design increase battery life on the wearable device. The system provides accurate physiological activity data to the needs of physicians for fall and in particular prefall analysis. The data base provides important information to analyze long-term trends and patterns needed in the prevention of fall. Comparing to [12], this system uses less power partly because the former using Bluetooth protocol instead of ZigBee. This system is also more versatile as the data are stored for fall analysis. Comparison studies were carried out for five different machine learning algorithms using data collected from the designed system. Naïve Bayes algorithm generated the best results with an accuracy of $97.3 \%$. The result suggests that among the algorithms tested, Naïve Bayes has the potential to perform better classification for the type of data used. Real-time classification algorithm is important for the fall detection device to achieve improved accuracy of the fall detection, which deserves further research.

\section{ACKNOWLEDGEMENTS}

The FANFARE project is funded by the Natural Sciences and Engineering Research Council (NSERC) of Canada under Strategic Project Grant number STPGP 350545.

\section{NOMENCLATURE}

Accelerometer $=$ a device for measuring acceleration and gravity induced reaction forces
Gyroscope

$=\mathrm{a}$ device for measuring or maintaining orientation, based on the principles of angular momentum

$g$

FANFARE $=$ Falls And Near Falls Assessment Research and Evaluation project

WEKA

= Waikato Environment for Knowledge Analysis: a popular suite of machine learning software written in Java, developed at the University of Waikato, New Zealand

ZigBee $\quad=$ a specification for a suite of high level communication protocols using small, low-power digital radios based on the IEEE 802.15.4-2006 standard for wireless personal area networks

\section{REFERENCES}

[1] L. Nyberg and Y. Gustafson, "Patient falls in stroke rehabilitation: A challenge to rehabilitation strategies," Stroke, vol. 26, pp. 838$842,1995$.

[2] J. Massion, "Postural control system," Curr. Opin. Neurobiol., vol. 4, no. 4, pp. 877-87, Dec. 1994

[3] D. Oliver, M. Britton, P. Seed, F. C. Martin, and A. H. Hopper, "Development and evaluation of evidence based risk assessment tool (STRATIFY) to predict which elderly inpatients will fall: Case control and cohort studies," BMJ, vol. 315, pp. 1049-1053, 1997.

[4] N. Noury, A. Fleury, P. Rumeau, A. K. Bourke, G. O. Laighin, V. Rialle, J. E. Lundy, "Fall detection - Principles and Methods," in Proceedings of the 29th Annual International Conference of the IEEE Engineering in Medicine and Biology Society (IEEE-EMBS), 2007, pp. 1663-166. 
[5] A. Newell and S. McKenna, "Activity monitoring for independent living using ceiling-mounted visual sensors," in International Conference on Aging, Disability and Independence, 2003.

[6] Sixsmith, N. Johnson, "A smart sensor to detect the falls of the elderly," IEEE Pervasive Comput., vol. 3, no. 2, pp. 42-47, AprilJune 2004.

[7] Najafi, K. Aminian, F. Loew, Y. Blanc, and P. A. Robert, "Measurement of stand-sit and sit-stand transitions using a miniature gyroscope and its application in fall risk evaluation in the elderly," IEEE Trans. Biomed. Eng., vol. 49, pp. 843- 851, Aug. 2002.

[8] Giansanti, "Investigation of fall-risk using a wearable device with accelerometers and rate gyroscope," Physiol. Meas., vol. 27, pp. 1081-1090, Nov. 2006.

[9] Y. Lee, J. Kim, M. Son, M. Lee, "Implementation of accelerometer sensor module and fall detection monitoring system based on wireless sensor network," in Proceedings of the 29th Annual International Conference of the IEEE Engineering in Medicine and Biology Society (IEEE-EMBS), 2007, pp. 2315-2318.

[10] JN5139: IEEE802.15.4 and ZigBee Wireless Microcontrollers, JN5139-PB v1.21, Jennic Ltd., 2008.

[11] M. J. Mathie, A. C. Coster, N. H. Lovell, and B. G. Celler, "Accelerometry: Providing an integrated, practical method for long term, ambulatory monitoring of human movement," Physiol. Meas., vol. 25, pp. R1-R20, 2004.

[12] J. Y. Hwang, J. M. Kang, Y. W. Jang, and H. C. Kim, "Development of novel algorithm and real-time monitoring ambulatory system using Bluetooth module for fall detection in the elderly," in Proceeding of the 26th Annual International Conference of the
IEEE Engineering in Medicine and Biology Society (IEMBS), 2004, vol. 3, pp. 2204-2207.

[13] S. Luo, and Q. Hu, "A dynamic motion pattern analysis approach to fall detection," The First IEEE International Workshop on Biomedical Circuits and Systems, 2004, pp. S2.1. 5-8

[14] M. Othman and T. Yau, "Comparison of different classification techniques using WEKA for breast cancer," in International Conference on Biomedical Engineering, vol. 15, 2007, pp. 520-523.

[15] WEKA at http://www.cs.waikato.ac.nz/ml/weka, September 3, 2008.

[16] J. Rodriguez, A. Goni, and A. Illarramendi, "Real-time classification of ECGS on a PDA," IEEE Transactions on Information Technology in Biomedicine, vol. 9, pp. 23-34, 2005.

[17] I. Witten and E. Frank, Data Mining: Practical Machine Learning Tools and Techniques, Morgan Kaufman, 2005.

[18] K. Cios, W. Pedrycz, R. Swiniarski, and L. Kurgan, Data Mining: A Knowledge Discovery Approach, Springer, 2007.

[19] H. Zhang and J. Su, "Naive Bayes for optimal ranking," J. Exp. Theor. Artif. Intell., vol. 20, pp. 79-93, 2008.

[20] S. Chakravarthy and J. Ghosh, "Scale-based clustering using the radial basis function network," Proceedings of IEEE International Conference on Neural Networks, 1994, pp. 897-902.

[21] C. Doukas, I. Maglogiannis, P. Tragas, D. Liapis, and G. Yovanof, "Patient fall detection using support vector machines," International Federation for Information Processing, 2007, vol. 247, pp 147-156.

(C) Dinh et al.; Licensee Bentham Open.

This is an open access article licensed under the terms of the Creative Commons Attribution Non-Commercial License (http://creativecommons.org/licenses/by-nc/3.0/) which permits unrestricted, non-commercial use, distribution and reproduction in any medium, provided the work is properly cited. 